\title{
Gastric Cancer, Linitis Plastica. EUS FNA Core Biopsy Technique as a Fast and Valuable Diagnostic Method Comparable to FNB
}

\section{Seyed Reza Fatemi ${ }^{1}$, Behzad Hatami ${ }^{1}$, Naghmeh Salarieh ${ }^{1 *}$, Azam Farahani ${ }^{1}$, Seyed Ali Fatemi ${ }^{2}$, Shahram Ebadi ${ }^{1}$ and Pardis Ketabi Moghadam ${ }^{1}$}

${ }^{1}$ Gastroenterology and Liver Diseases Research Center, Research Institute for Gastroenterology and Liver Diseases, Shahid Beheshti University of Medical Sciences, Tehran, Iran

${ }^{2}$ Life Science, Biology, Student, University of Toronto, Canada

*Corresponding Author: Naghmeh Salarieh, Gastroenterology and Liver Diseases Research Center, Research Institute for Gastroenterology and Liver Diseases, Shahid Beheshti University of Medical Sciences, Tehran, Iran.
Received: August 05, 2021

Published: 00-00

(C) All rights are reserved by Naghmeh Salarieh., et al.

\section{Abstract}

A fifty-year-old man was referred with progressive abdominal pain accompanying with anorexia and weight loss. Upper endoscopy was considered as a helpful procedure for the patient.

Upper endoscopy revealed increased mucosal fold thickness due to decreased expansion in body and fundus with multiple erosions, in favor of mural infiltration compatible with diffuse type of gastric cancer, multiple standard biopsies were taken but pathology was reported negative. Abdominal CT-SCAN was done and it was highly suspicious of malignancy, so bite on bite biopsies were performed again. Pathology report was negative for the second time. Eventually, EUS-FNA was done, full thickness of all layers of stomach with multiple lymph nodes were noticed in EUS. With 22 G needle, FNA with slow-pull and multiple pass technique was performed to obtain many and sufficient core samples for cellblock cytology was taken. Pathology report was adenocarcinoma with signet ring cells.as a result, EUS-FNA was an accurate procedure for diagnosing gastric cancer.

Keyword: EUS FNA; EUS FNB; Linitis Plastica; Gastric Cancer

\section{Introduction and Case Report}

The patient was a fifty-year-old man with chief complaint of abdominal pain, progressive anorexia and weight loss in the last three months before referring to our hospital. Abdominal dull pain with mild back radiation was the main problem.

On endoscopy, abnormally diffused thickening of gastric folds in the body and fundus with superficial ulcers and erosions were noticed. Gastric expansion was intensively decreased and endo- scopic features were suggestive of infiltrative gastric cancer. Multiple standard biopsies were taken but pathology only revealed inflammation. medical treatment for Helicobacter pylori eradication was done but symptoms were continued so abdominal CT scan was requested and due to increased mural thickness, malignancy was still one of important differential diagnoses This time, bite on bite biopsies were taken during endoscopy, but for the second time histologic findings were negative for malignancy (Figure 1). 


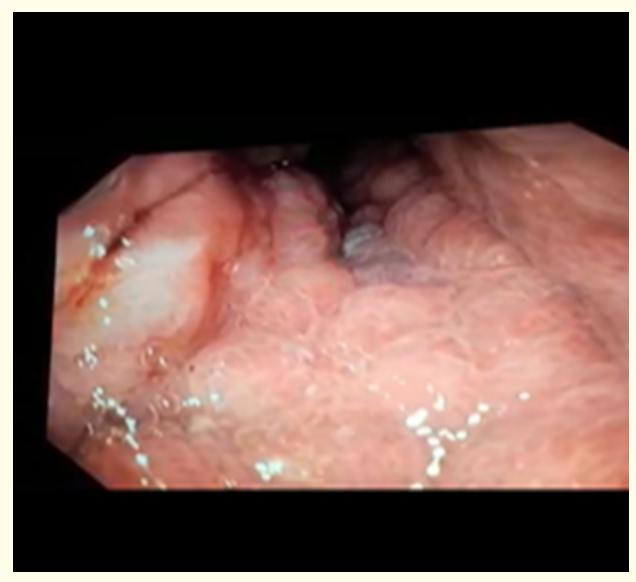

Figure 1: Endoscopic appearance.

According to high index of suspicion, EUS was considered as next diagnosing step. On endosonography, obvious wall thickening was noted in second to fourth gastric layers with some surrounding lymph nodes. Needle gauge 22 was inserted and multiple biopsies from different directions with multipass method were obtained. Core needle biopsies were taken with slow-pull (capillary) method and samples were sent to pathology for cell-block cytology. Histologic findings revealed adenocarcinoma with signet ring cells. The patient was referred to oncologist for further treatment. This experience showed that in patients suspicious of linitis plastica EUS is an effective, fast and accurate diagnostic procedure (Figure 2-4).

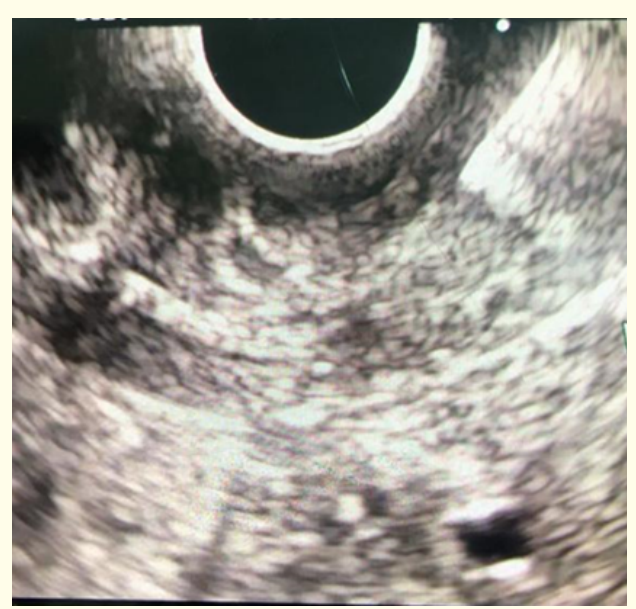

Figure 2: Wall thickness $=7 \mathrm{MM}$.

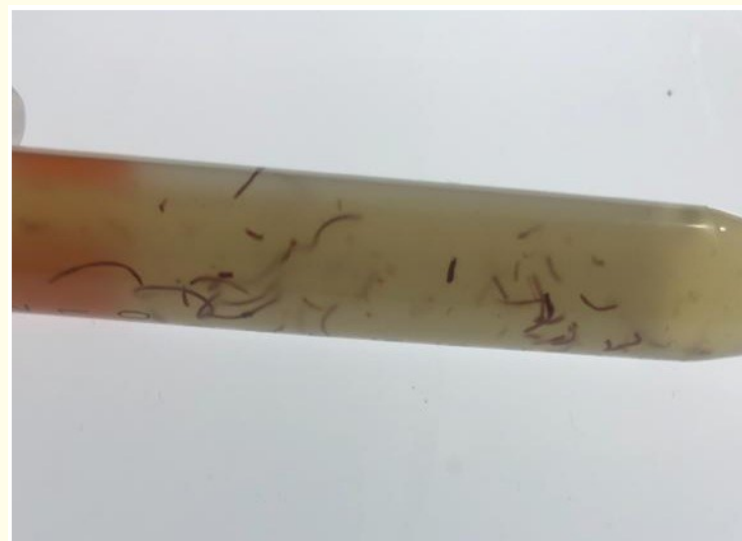

Figure 3

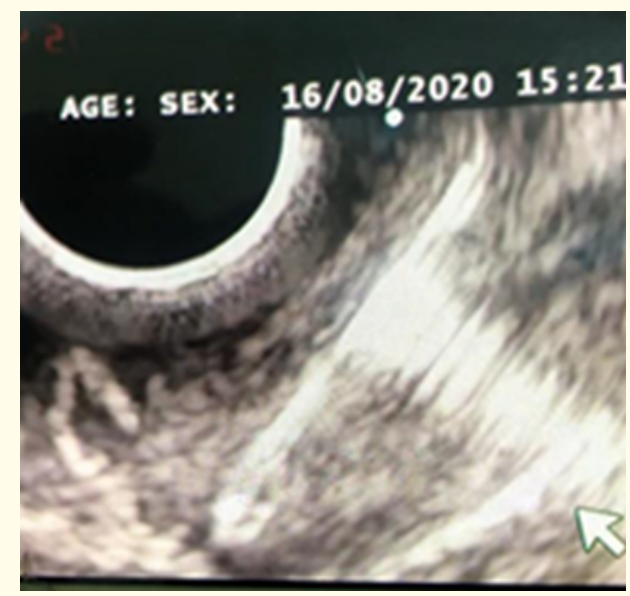

Figure 4

\section{Discussion}

Diffuse or infiltrative type of gastric adenocarcinoma extend across stomach due to mutation of CDH1 gene or E-cadherin and loss of ICAM1 protein function. This type of gastric cancer usually is diagnosed when expansion of stomach is decreased significantly [1].

Linitis plastica, in contrast intestinal type of gastric cancer does not present with solitary gastric mass. Two different subtypes of Linitis Plastica have been described. In first type, giant folds or waffle-like mucosa is noticed. The first type starts from the proxi- 
mal part of stomach and extends to the great curvature. The second type originates from the antrum and is shown as an increase in the layers thickness and atrophy of the mucosa [2].

Difficult diagnosis of linitis plastica by regular biopsies cause a delay in starting treatment, next diagnosing steps like bite-on-bite biopsies and EMR method for obtaining biopsies occasionally report negative results. Studies have shown high rate of non-diagnostic biopsies (30\% - 36\%) [3].

There is no gold standard method for diagnosing Linitis Plastica, in case of strong clinical suspicion, sampling should be repeated in different ways despite negative tissue. On EUS, depth, expansion and staging of cancer as well as access to deep layers are possible and according to the type and axis of the layer involved in differentiating malignant lesions from benign is helpful $[5,6]$.

Yingjian., et al. studied 46 patients who were suspected of gastric Linitis Plastica and endoscopic biopsies showed negative results, and they underwent EUS-FNA. The diagnostic accuracy EUSFNA was $87 \%$ [7].

EUS-FNB can be a detailed procedure that is done with needles which have crow or fork like tips for obtaining samples, although due to better access to FNA needles, EUS-FNA can be done more frequently.

Due to the limitations of the FNA, needles designed to obtain biopsy specimens (Fine-needle-biopsy [FNB] needles) have been developed to allow core samples collecting [8].

The available literature comparing FNA and FNB needles has not provided definitive results [9].

A recent metanalysis found no significant difference between one biopsy needle (Procore, cook endoscopy) and standard FNA needles with regard to sample adequacy. However, the FNB needle established a diagnosis with different passes [10].

In our case, sufficient samples were obtained for diagnosis through FNA.

\section{Conclusion}

As a result, taking core samples through EUS_FNA is fast and accurate method as FNB technique in Linitis Plastica type of gastric cancer.

\section{Bibliography}

1. AP Carneiro., et al. "E-cadherin dysfunction in gastric cancer - Cellular consequences, clinical applications and open questions". FEBS Letters 586.18 (2012): 2981-2989.

2. K Jung., et al. "Borrmann type 4 advanced gastric cancer: focus on the development of scirrhous gastric cancer". Clinical Endoscopy 49 (2016): 336-345.

3. M Rahman. "Linitis Plastica of The Stomach: A Review". Journal of Surgical Sciences 22.2 (2018): 125-138.

4. T Rice., et al. "Esophageal ultrasound and the preoperative staging of carcinoma of the esophagus". Journal of Thoracic and Cardiovascular Surgery 101.3 (1991): 536-543.

5. M Levine., et al. "Scirrhous carcinoma of the stomach: radiologic and endoscopic diagnosis". Radiology 175.1 (1990): 151154.

6. N Kalantzis., et al. "The role of endoscopic ultrasonography in diagnosis of benign lesions of the upper GI tract". European Journal of Surgical Oncology 19.5 (1993): 449-454.

7. YE Yingjian and T Shiyan. "Endoscopic ultrasound guided fine needle aspiration biopsy for diagnosis of gastric linitis plastica with negative malignant endoscopy biopsies". Oncology Letters 16.4 (2018): 4915-4920.

8. L MJ and W MJ. "EUS-guided Trucut biopsy". Gastrointestinal Endoscopy 62.3 (2005): 417-426.

9. S DS., et al. "EUS-guided 22-gauge fine-needle aspiration versus core biopsy needle iatic neoplasms". Diagnostic Cytopathology 42.9 (2014): 751-758.

10. B JY., et al. "A meta-analysis comparing ProCore and standard fine-needle aspiration needles for endoscopic ultrasoundguided tissue acquisition". Endoscopy 48.4 (2015): 339-349.

\section{Volume 4 Issue 9 September 2021 C) All rights are reserved by Naghmeh Salarieh., et al.}

